\title{
Preface to special issue in honor of Tomasz Kapitaniak's 60th birthday: nonlinear and complex physics
}

\author{
Yuri Maistrenko ${ }^{1, a}$, Przemyslaw Perlikowski ${ }^{2, b}$, and Serhiy Yanchuk ${ }^{3, c}$ \\ ${ }^{1}$ Institute of Mathematics and Centre for Medical and Biotechnical Research, National \\ Academy of Sciences of Ukraine, Tereshchenkivska St. 3, 01030 Kyiv, Ukraine \\ 2 Division of Dynamics, Lodz University of Technology, Stefanowskiego 1/15, \\ 90-924 Lodz, Poland \\ ${ }^{3}$ Institute of Mathematics, Technische Universität Berlin, Straße des 17. Juni 136, \\ 10623 Berlin, Germany
}

Received 4 September 2020 / Accepted 4 September 2020

Published online 28 September 2020

This edition is one of two special issues dedicated to Tomasz Kapitaniak on the occasion of his 60th birthday in recognition of his outstanding contributions in Nonlinear Science and Theoretical Mechanics. The second celebrating issue will appear soon in Meccanica. Tomasz Kapitaniak is currently a Professor at the Faculty of Mechanical Engineering, Lodz University of Technology. He is also a real member of the Polish Academy of Sciences. The present edition makes an emphasis on the Tomasz' research activity in the field of Network Dynamics and Chimera states - a modern, challenging direction of nonlinear science. The issue presents fourteen papers that cover different aspects of the theory and applications. We hope they will serve the scientific community as a reference in many future research studies.

About half of the contributions to the special issue deals with the chimera states, a rapidly growing branch at the border of nonlinear dynamics and statistical physics in application to Network Science. The remaining articles develop a different aspect of Nonlinear and Complex Physics, from Theory to Application. Below we give a short characteristic of results obtained in each of them.

Balcerzak et al. [1] study synchronization between identical systems coupled unidirectionally with a master oscillator of the same type. Two class of systems are taken into account in the paper, i.e., given by smooth and non-smooth ordinary differential equations. The authors show that with the increase of the coupling parameter, a generalized synchronization (GS) occurs. First, the oscillators perform independent motion then they start to synchronize in the weak GS regime, and finally, they reach the strong GS state. These transitions are visible in the spectrum of Lyapunov exponents. Korneev et al. [5] consider a non-autonomous memristor-based oscillator with a line of equilibria driven by a periodic force. Two synchronization mechanisms are presented: the capture of the phase and frequency of the oscillator, and suppression of oscillations by an external signal. The investigations of the stability of a 3 -torus solution in a ring of unidirectionally coupled Duffing oscillators are presented by

\footnotetext{
a e-mail: y.maistrenko@biomed.kiev.ua

b e-mail: przemyslaw.perlikowski@p.lodz.pl

c e-mail: yanchuk@math.tu-berlin.de
} 
Borkowski et al. [8]. The solution stabilizes due to the appearance of a superposition of two independent effects characterized with irrational frequencies, i.e., the classical Newhouse, Ruelle and Takens scenario, and rotating wave flow typical for such type of coupling.

The synchronization in a non-local ring network of adaptively coupled phase oscillators is considered by Berner et al. [3]. This paper includes a description of a variety of frequency synchronized states such as phase-locked, multicluster and solitary states, supported with rigorous stability analysis. The explanation of the emergence of solitary states and classification of several bifurcation scenarios in which these states are created and stabilized are provided.

The stability of systems with discontinuities utilizing Lyapunov exponents is studied by Balcerzak et al. [2]. They show a fast and simple method of estimation of exponents and illustrate it with an externally forced system impacting in stop. The presented algorithm can simplify investigations of non-smooth dynamical systems. Pei and Jia [10] study an active control system with state-dependent actuation time delay. The complexity of the system is reduced to a low-dimensional approximation using the Galerkin method. Stability and Hopf bifurcation are investigated by the Routh-Hurwitz criterion. All analytical results are confirmed with numerical simulations.

Wang et al. [14] focus on a recently discovered type of multistable systems called megastable. Such systems have infinite number of coexisting attractors. In type 1 megastability, the number of attractors reflects the number of equilibria, and in type 2 there is no such relation. The authors introduce a first megastable system which has a singularity in its equations. Using standard tools of analysis, they show the reach variety of system dynamics, including limit cycle, torus, and strange attractor. Another engineering application, the highly precise model of the kinematics of the motorcycle, is presented by Lazarek et al. [6]. The introduced iterative method allows calculating a proper pitch angle value and position of the front wheel tyre-ground contact point. The dependence between the capsize mode, and the pitching behaviour is clearly stated, and straightforward application of the algorithm to the electronic control units protecting from wheelie phenomena is described.

The chimera state is a phenomenon in the nonlinear coupled systems known since 2002. It is characterized by the co-existence of synchronous and non-synchronous clusters in a homogenously coupled network. Lu et al. [7] study a network of nonidentical coupled van der Pol's oscillators and investigate the robustness of chimera states against the oscillator's frequency mismatches. It is shown that, in the case of non-identical systems, the chimera state passes to complete synchronization state for a higher value of the coupling strength than in case of identical coupled systems. Moreover, the observed chimeras are mostly non-stationary.

The metamorphoses of spiral wave chimera states in coupled phase oscillators with inertia are reported by Maistrenko et al. [13]. The unusual behavior of the system occurs, when the coupling parameter enters the so-called solitary region. In this range, isolated oscillators are present on the spiral core background of the chimera, and they start to oscillate with different time-averaged frequencies (Poincare winding numbers). Paper of Ebrahimzadeh et al. [4] is devoted to the study of chimera states in the smallest possible network consisting of three metronomes with phase-lagged all-toall coupling. The theoretical investigations are supported by a laboratory experiment. The chimera states manifest themselves as a mismatch of average frequency between two synchronous and one desynchronized oscillator. An ensemble of nonlocally coupled excitable FitzHugh-Nagumo systems with noise is studied by Semenova [11]. The addition of noise to the system results in the appearance of chimera states called coherence-resonance chimera. This is the first observation of such a phenomenon in excitable systems. The article by Varshney et al. [12] is devoted to chimera-like states 
in a network of driven FitzHugh-Nagumo oscillators with time-dependent slowly varying parameter. Near the bifurcation point, it leads to the slow-passage effect and a time delay in the bifurcation. Moreover, when the symmetry of coupling is broken (conjugate coupling), one observes the appearance of chimera-like structures.

In contrast to previously discussed papers, Ouannas et al. [9] study the dynamics of the discrete-time system given by a fractional form of Henon-Lozi type map. Authors show that the general behavior of the analyzed map depends on the fractional-order, and they introduce control law to stabilize the states of the system.

In summary, this special issue manifests a broad spectrum of current studies on nonlinear dynamics, network theory, and complex systems focused on applications in physics and engineering. We hope that this collection of papers reflects the diversity of present research directions in this area and that the compilation of recent advances is indeed timely. We wish to thank all contributing authors for submitting their articles. Since this edition is also a Festschrift on the occasion of Tomasz Kapitaniak's 60th birthday, the circle of the contributors includes a number of long-term Tomasz's collaborators working presently in different countries throughout the world, and many contributors address directly to Tomasz's results.

Happy Birthday, Tomasz!

\section{References}

1. M. Balcerzak, A. Chudzik, A. Stefanski, Eur. Phys. J. Special Topics 229, 2151 (2020)

2. M. Balcerzak, T. Sagan, A. Dabrowski, A. Stefanski, Eur. Phys. J. Special Topics 229, 2167 (2020)

3. R. Berner, A. Polanska, E. Schöll, S. Yanchuk, Eur. Phys. J. Special Topics 229, 2183 (2020)

4. P. Ebrahimzadeh, M. Schiek, P. Jaros, T. Kapitaniak, S. van Waasen, Y. Maistrenko, Eur. Phys. J. Special Topics 229, 2205 (2020)

5. I.A. Korneev, A.V. Slepnev, V.V. Semenov, T.E. Vadivasova, Eur. Phys. J. Special Topics 229, 2215 (2020)

6. M. Lazarek, J. Grabski, P. Perlikowski, Eur. Phys. J. Special Topics 229, 2225 (2020)

7. H. Lu, F. Parastesh, A. Dabrowski, H. Azarnoush, S. Jafari, Eur. Phys. J. Special Topics 229, 2239 (2020)

8. L. Borkowski, A. Stefanski, Eur. Phys. J. Special Topics 229, 2249 (2020)

9. A. Ouannas, A.-A. Khennaoui, X. Wang, V.-T. Pham, S. Boulaaras, S. Momani, Eur. Phys. J. Special Topics 229, 2261 (2020)

10. L. Pei, H. Jia, Eur. Phys. J. Special Topics 229, 2275 (2020)

11. N. Semenova, Eur. Phys. J. Special Topics 229, 2295 (2020)

12. V. Varshney, S. Kumarasamy, B. Biswal, A. Prasad, Eur. Phys. J. Special Topics 229 2307, (2020)

13. V. Maistrenko, O. Sudakov, Y. Maistrenko, Eur. Phys. J. Special Topics 229, 2327 (2020)

14. Z. Wang, H.R. Abdolmohammadi, M. Chen, A. Chudzik, S. Jafari, I. Hussain, Eur. Phys. J. Special Topics 229, 2341 (2020) 